\title{
Selective Expression of $\beta$ Tubulin Isotypes in Gerbil Vestibular Sensory Epithelia and Neurons
}

\author{
Brian Perry, ${ }^{1}$ Heather C. Jensen-Smith, ${ }^{2}$ Richard F. Ludueña, ${ }^{3}$ and Richard Hallworth ${ }^{2}$ \\ ${ }^{1}$ Department of Pediatrics, The University of Texas Health Science Center at San Antonio, San Antonio, TX 78229-3900, USA \\ ${ }^{2}$ Department of Biomedical Sciences, Creighton University, Omaha, NE 68178, USA \\ ${ }^{3}$ Department of Biochemistry, The University of Texas Health Science Center at San Antonio, San Antonio, \\ TX 78229-3900, USA
}

Received: 18 October 2001; Accepted: 13 December 2002; Online publication: 10 February 2003

\section{ABSTRACT}

The seven mammalian isotypes of $\beta$ tubulin are strikingly similar in amino acid sequence. The differences in isotypic sequence, although small, are nonetheless conserved in evolution, which suggests that they may confer distinct functional roles. If so, such roles should be reflected in the selective expression of isotypes by cell type, or even in the sorting of isotypes to within-cell pools. Hair cells of the vestibular sensory epithelia each possess a kinocilium, a microtubule-based organelle that could represent a distinct microtubule compartment, separate from the extensive microtubule network in the soma. The afferent neurons that innervate the vestibular sensory epithelia may also be functionally divided into dendritic, somatic, and axonal compartments, each with its own complement of microtubules. We have examined the distribution of $\beta$ tubulin isotypes in gerbil vestibular epithelia using isotype-specific antibodies to four isotypes and indirect immunofluorescence. We found that hair cells selectively express $\beta_{\mathrm{I}}$ and $\beta_{\mathrm{IV}}$ tubulin, while supporting cells express $\beta_{\mathrm{I}}, \beta_{\mathrm{II}}$, and $\beta_{\mathrm{IV}}$ tubulin. However, no sorting of isotypes between somatic and kinocilia compartments was found in hair cells. Vestibular ganglion cells display three isotypes in the soma, axon, and terminal dendrite compartments $\left(\beta_{\mathrm{I}}, \beta_{\mathrm{II}}\right.$, and $\beta_{\mathrm{III}}$ tubulin), but only $\beta_{\mathrm{III}}$ tubulin was found in calyceal nerve endings. The

Correspondence to: Richard Hallworth - Department of Biomedical Sciences - Creighton University - 2500 California Plaza - Omaha, NE 68178. Telephone: (402) 280-3057; fax: (402) 280-2690; email: hallw@creighton.edu implication of these findings is that $\beta$ tubulin isotypes are not sorted to within-cell compartments in hair cells but are sorted in some vestibular neurons.

Keywords: vestibular sensory epithelia, hair cell, supporting cell, ganglion cell, $\beta$ tubulin, tubulin isotypes

\section{INTRODUCTION}

Microtubules consist of heterodimers of $\alpha$ and $\beta$ tubulin, both of which exist as numerous isotypes. Seven isotypes of mammalian $\beta$ tubulin have been characterized, termed $\beta_{\mathrm{I}}, \beta_{\mathrm{II}}, \beta_{\mathrm{III}}, \beta_{\mathrm{IVa}}, \beta_{\mathrm{IVb}}, \beta_{\mathrm{V}}$, and $\beta_{\mathrm{VI}}$ (Ludueña 1998). With the exception of $\beta_{\mathrm{VI}}$, the $\beta$ tubulin isotypes are among the most highly conserved proteins known (Ludueña 1998). The multi-tubulin hypothesis (Fulton and Simpson 1976) proposed that different forms of tubulin could mediate the different functional roles of microtubules. A prediction of this hypothesis is that the isotypes will be selectively expressed in different tissues and may even be compartmentalized within cells according to function. Consistent with the hypothesis, it has been found that different isotypes are expressed in different cell types of the same tissue (Roach et al. 1998; Hallworth and Ludueña 2000). However, little is known about the functional roles of $\beta$ tubulin isotypes. An exception to this statement is that $\beta_{\mathrm{IV}}$ tubulin appears to be an important component of axonemal microtubules (Renthal et al. 1993; Lu et al. 1998; Roach et al. 1998) and may be associated with actin stress fibers in certain cells (Walss-Bass et al. 2001). 
Vestibular sensory epithelia consist of sensory hair cells, supporting cells, afferent neurons, and efferent nerve endings. The apical surfaces of hair cells each bear multiple actin-based stereocilia and a single polarized kinocilium, a microtubule-based organelle. The kinocilium has the $9+2$ paired organization of microtubules characteristic of the axoneme, but it is not motile. Other somatic microtubules in hair cells originate at the cuticular plate or nucleating centers near the epithelial apex (Jaeger et al. 1994) and likely have roles in intracellular trafficking and in the maintenance of cell shape. Each hair cell makes synaptic contact with the dendrites of one or more afferent neurons. Vestibular sensory epithelia are also extensively innervated by neurons of the vestibular ganglion. The microtubules of the afferent neurons whose dendrites innervate the vestibular epithelia may also be functionally divided into dendritic, somatic, and axonal compartments. The vestibular sensory epithelia are therefore an interesting test of the possibility of selective expression and cellular sorting of $\beta$ tubulin isotypes.

In this study we examined the distribution of $\beta$ tubulin isotypes in vestibular sensory epithelia and neurons of the gerbil. Isotypes were detected using four isotype-specific antibodies directed against $\beta_{\mathrm{I}}$, $\beta_{\mathrm{II}}, \beta_{\mathrm{III}}$, and $\beta_{\mathrm{IV}}$ tubulin raised in mouse (the only such comprehensive panel of antibodies available; Roach et al. 1998) and indirect immunofluorescence. The antibody against $\beta_{\mathrm{IV}}$ tubulin is unable to distinguish between $\beta_{\mathrm{IVa}}$ and $\beta_{\mathrm{IVb}}$, which differ by only two residues (Ludueña 1998). We have used a variety of approaches to preparation of tissue for examination, including frozen sections, whole mounts, and dissociation of fresh sensory organs into isolated cells.

We found that hair cells express only $\beta_{\mathrm{I}}$ and $\beta_{\mathrm{IV}}$ tubulin, while supporting cells express $\beta_{\mathrm{I}}, \beta_{\mathrm{II}}$, and $\beta_{\mathrm{IV}}$ tubulin. In hair cells, the same isotypes were observed in both somatic and kinocilia compartments. Vestibular ganglion cells express three isotypes, which are found in the soma, the axon, and the dendrite $\left(\beta_{\mathrm{I}}\right.$, $\beta_{\mathrm{II}}$, and $\left.\beta_{\mathrm{III}}\right)$. The same three isotypes were found in the Schwann cell investments of peripheral dendrites. However, only $\beta_{\mathrm{III}}$ tubulin was found in calyceal nerve endings. Calyceal nerve endings the large dendritic terminations of a subpopulation of vestibular ganglion afferents. Thus, at least some vestibular ganglion cells both selectively express $\beta$ tubulin isotypes and sort them into compartments.

\section{MATERIALS AND METHODS}

The distribution of $\beta$ tubulin isotypes was examined in adult gerbil vestibular epithelia using indirect immunofluorescence in frozen sections, whole mounts, and dissociated cells. Adult gerbils (20 days old or older) were anesthetized with Nembutal and cardiacperfused with $10-15 \mathrm{ml}$ of a solution of $4 \%$ paraformaldehyde dissolved in phosphate buffered saline (PBS). For whole mounts and sections, temporal bones were removed from the head, postfixed for $2 \mathrm{~h}$, and decalcified in EDTA. For whole mounts, individual macula and ampullary organs were dissected out and processed for immunolabeling as described below. In whole mounts and sections of dissected organs, we were generally unable to distinguish the subtypes of organ classes (utricular and saccular maculae, horizontal, superior and posterior cristae), but we have indicated the subtype where known. Most macula organs were utricles. We saw no differences in the labeling patterns across organ types. Occasionally, segments of vestibular nerve, with macula and crista organs attached, were processed as whole mounts.

For sections, one of two methods was employed: either individual sensory organs were dissected out from decalcified temporal bones, or decalcified temporal bones were embedded in agar and bisected with a razor blade. The organs or halves were then processed to equilibration in $30 \%$ sucrose in PBS as a cryoprotectant and were then quickly frozen in O.C.T. compound (Miles Labs, Elkart, IN) using methanol cooled by dry ice. Eight or ten micron sections were cut on a cryostat (Leica Microsystems, Bannockburn, IL). Whole mount preparations and sections were blocked and permeabilized in PBS containing $1 \%$ bovine serum albumin (BSA), $0.25 \%$ Triton-X, and $5 \%$ normal goat serum. The presence of $\beta$ tubulin isotypes was detected with monoclonal antibodies to $\beta_{\mathrm{I}}, \beta_{\mathrm{II}}, \beta_{\mathrm{III}}$, or $\beta_{\mathrm{IV}}$, tubulin raised in mouse as previously described (Banerjee et al. 1988, 1990, 1992; Roach et al. 1998). Each antibody was prepared to an epitope unique to the C-terminus of that isotype. The C-termini of $\beta_{\mathrm{IVa}}$ and $\beta_{\mathrm{IVb}}$ are virtually identical, and therefore the anti- $\beta_{\text {IV }}$ antibody was unable to discriminate between them. The primary antibody was made visible by staining with goat anti-mouse IgG coupled to fluorescein isothiocyanate (Sigma, St. Louis, MO) or to Alexa 488 (Molecular Probes, Eugene, OR). The chicken $\beta_{\text {III }}$ antibody TUJ1 raised in mouse (Lee et al. 1990) was obtained from Research Diagnostics Inc. (Flanders, NJ), and the same goat anti-mouse secondary antibodies were used. A specific antibody to myosin VIIa (Hasson et al. 1995), raised in rabbit, was detected using antibodies to rabbit IgG raised in donkey and coupled to Cy3 (Jackson ImmunoResearch, West Grove, PA). Polymerized actin was detected using phalloidin conjugated to Alexa 568 (Molecular Probes). Sections and whole mounts were sealed under coverslips in 50\% PBS: 50\% glycerol containing $1 \% n$-propylgallate as an antifade agent. Negative controls were performed by omitting the 
primary antibody. Positive controls were performed using the same procedures as above with a monoclonal primary antibody to $\beta$ tubulin raised in mouse (Sigma, clone TUB 2.1; Gozes and Barnstable 1982). This antibody has previously been shown to bind to all known populations of microtubules in the gerbil cochlea (Hallworth et al. 2000). Similarly, all features of the labeling patterns described in this article have been observed using this antibody.

Isolated vestibular cells were prepared as follows: A day prior to the experiment, \#1 coverslips were coated in Concanavalin A (Con A; Sigma) as described by Maue and Dionne (1987) and stored in a humidified atmosphere. Adult gerbils (20 days old or older) were anesthetized with Nembutal and decapitated. Vestibular epithelia were quickly dissected from the temporal bones and placed in low-calcium saline containing $1 \%$ papain (Calbiochem, La Jolla, CA). The solution was gently agitated for $30 \mathrm{~min}$, after which the enzyme solution was removed and replaced with saline $(0.9 \%$ $\mathrm{NaCl}$ ) containing $0.001 \%$ DNAase I (Sigma). After 5 min, the mixture was then triturated until turbid using a syringe. Cells from the resulting suspension were plated on the Con A-coated coverslips, which were maintained in a humidified chamber at room temperature. After allowing $30 \mathrm{~min}$ for the cells to attach, the cells were fixed in PBS containing $0.25 \%$ paraformaldehyde for $10 \mathrm{~min}$. The fixed cells were then processed for immunolabeling essentially as above.

Specimens were photographed using an Axioskop II microscope (Carl Zeiss Jena, Jena, Germany) equipped with $40 \mathrm{X}$ and 100X objectives. Images were obtained using a Spot RT digital camera (Diagnostic Instruments, Sterling Heights, MI) and were analyzed and prepared for presentation using Photoshop (Adobe Systems, San Jose, CA). Confocal images were obtained on a Radiance 2000 confocal microscope (Bio-Rad, Hercules, CA) on a Nikon Eclipse 800 upright microscope equipped for epifluorescence (Nikon Instruments, Melville, NY).

For immunoblotting, vestibular epithelia removed from anesthetized gerbils were homogenized and a uniform amount of protein $(18 \mu \mathrm{g})$ was loaded into each lane of a $10 \%$ polyacrylamide gel (Gradipore, French's Forest, New South Wales, Australia). The protein content of each sample was determined by a protein concentration assay using a microplate reader (Titertek, Thermo Labsystems, Finland). The lanes were run at $150 \mathrm{~V}$ for $90 \mathrm{~min}$ in sodium dodecyl sulfate electrophoresis buffer. The proteins were then transferred from the gels to nitrocellulose sheets (100 $\mathrm{V}$ for $1 \mathrm{~h}$ ). The protein lanes were cut into strips for antibody labeling and were exposed to one of the five primary antibodies (four $\beta$ tubulin isotype-specific antibodies plus the $\beta$ tubulin monoclonal antibody described earlier) overnight at $4^{\circ} \mathrm{C}$. After rinsing, the

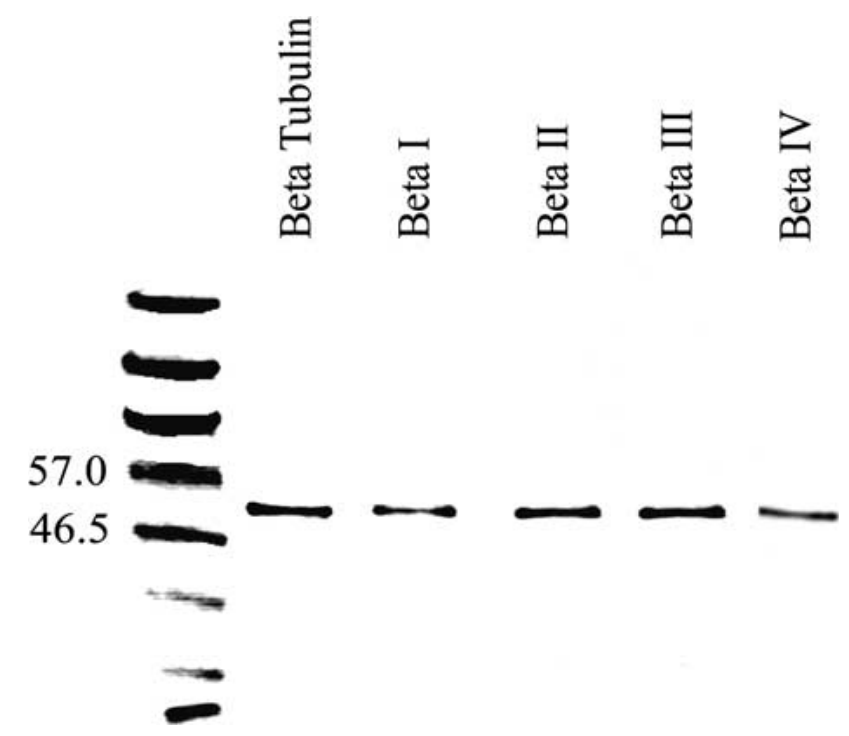

FIG. 1. Western blots showing immunolabeling of gerbil vestibular epithelia with an antibody to $\beta$ tubulin (second lane from left) and the isotype-specific anti- $\beta$ tubulin antibodies used in this study (lanes 3 through 6). A protein ladder with the molecular weights indicated is in the leftmost lane.

protein strips were incubated in anti-mouse IgG linked to biotin (Cell Signaling Technology, Beverly, MA) for $1 \mathrm{~h}$ at room temperature. The markers were labeled with horseradish peroxidase-conjugated antibiotin antibodies (Cell Signaling Technology). The protein strips were rinsed again and treated for 10 min with Luminol reagent (Santa Cruz Biotechnology, Santa Cruz, CA) and exposed to XAR5 scientific imaging film (Kodak, Rochester, NY).

Animal care and handling was performed in conformance with approved protocols of the University of Texas Health Science Center at San Antonio and Creighton University School of Medicine Institutional Animal Care and Use Committees.

\section{RESULTS}

\section{Immunoblots}

Western blots of vestibular sensory epithelia indicated that labeling for all $\beta$ tubulin isotypes in vestibular tissue is restricted to a single band of molecular weight close to $50 \mathrm{kDa}$ (Fig. 1). The molecular weight of $\beta$ tubulin is $50 \mathrm{kDa}$ (Ludueña 1998). The amino acid sequence differences between isotypes are so small that the isotypes are indistinguishable by molecular weight alone.

\section{Identification of cell types in vestibular organs}

Microtubules are present in vestibular hair cells, supporting cells, and innervating both macula and 

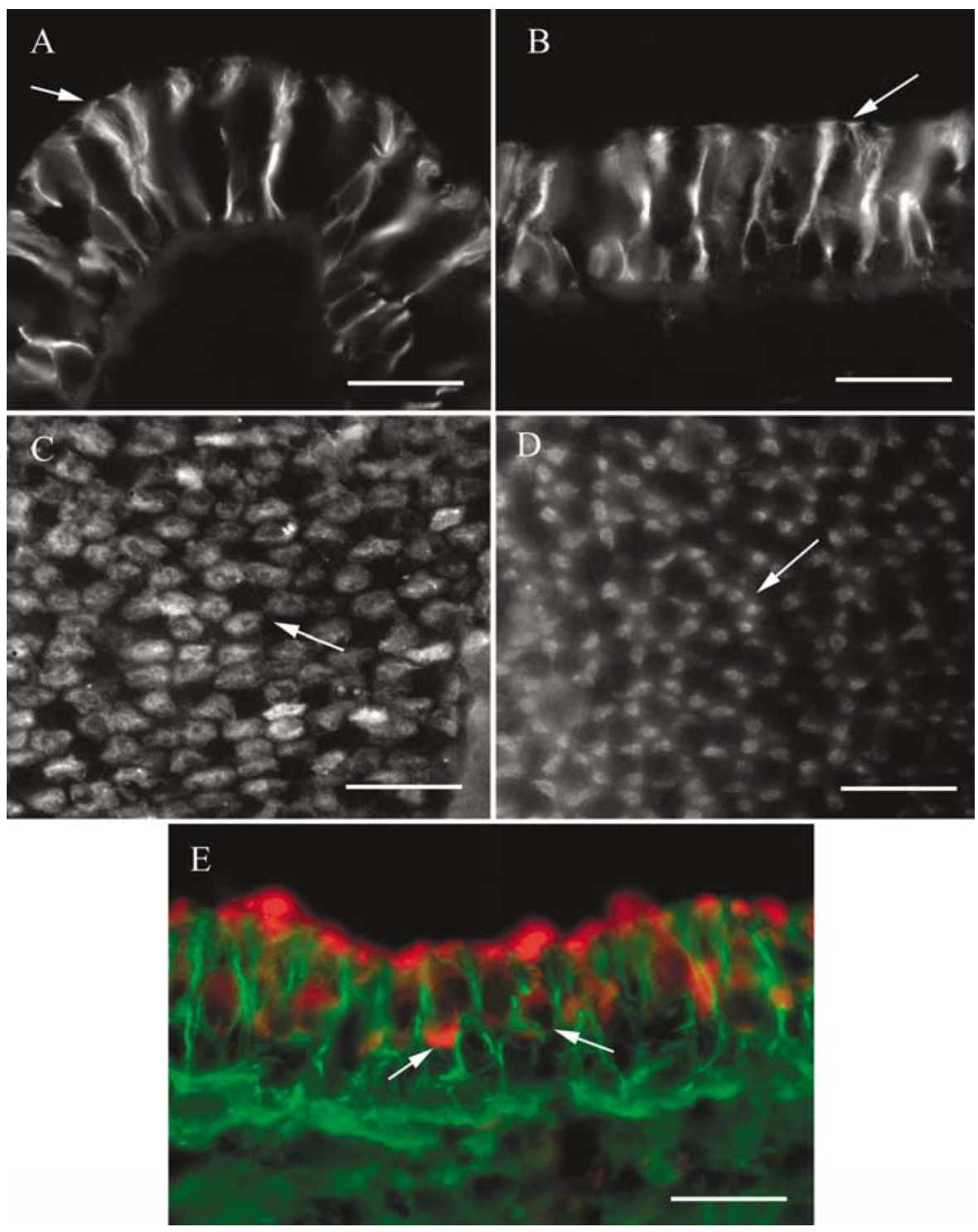

FIG. 2. $\quad \beta_{\text {II }}$ tubulin in vestibular epithelia. A Transverse section of a crista ampullaris showing label in supporting cells. Note the large tufts at the apical ends (arrow). B Apical view of a transverse section of a macula. The apical tufts in the supporting cells (arrow) are smaller than those in crista organs. C Apical view of a crista ampullaris whole mount showing the label patches in four or five supporting cells in a cluster surrounding an unlabeled area. A typical cluster is indicated by the arrow. D Whole mount of a utricular macula showing clusters of label patches in the five to seven presumed supporting cells surrounding an unlabeled area. A typical cluster is indicated by the arrow. $\mathbf{E}$. Transverse section of a macula labeled for $\beta_{\text {II }}$ tubulin (green) and myosin VIla (red). Label for myosin VIla in two hair cells is indicated by the arrows. The scale bar represents $20 \mu \mathrm{m}$ for all panels. ampullary organs. Hair cells were identified by nuclei that were central in the epithelium and by the concentration of microtubules in the supranuclear region. Supporting cells had nuclei located in the base of the epithelium and exhibited a microtubule bundle that spanned the entire thickness of the epithelium (Ogata and Slepecky 1995).

We describe our findings by isotype, starting with the simplest pattern, that for $\beta_{\text {II }}$ tubulin.

$\boldsymbol{\beta}_{\text {III }}$. Label for $\beta_{\text {II }}$ tubulin in vestibular sensory epithelia consisted only of the microtubules in the perinuclear region and the processes of supporting cells. For example, Figure 2A shows a section of a crista ampullaris labeled for $\beta_{\text {II }}$ tubulin. The labeling pattern consists of strands surrounding the supporting cell nuclei and spanning the sensory epithelium, consistent with the appearance of supporting cell microtubules. The apical ends of these strands possessed large tufts. In sections of maculae (Fig. 2B), the labeling pattern was similar but the apical tufts were much smaller.

In whole mounts, focusing at the apical surface of a crista, a characteristic pattern of irregular round patches of label was observed (Fig. 2C), presumed to be the apical tufts of supporting cells. These patches were clustered in groups of four or five around a central unlabeled area corresponding to the expected position of a hair cell. In whole mounts of maculae (Fig. 2D), the patches of label were smaller, corresponding to the smaller tufts observed in sections, and were usually elongated instead of round. The patches were also arranged in circular clusters of five to seven surrounding an unlabeled area where a hair cell would be expected. 

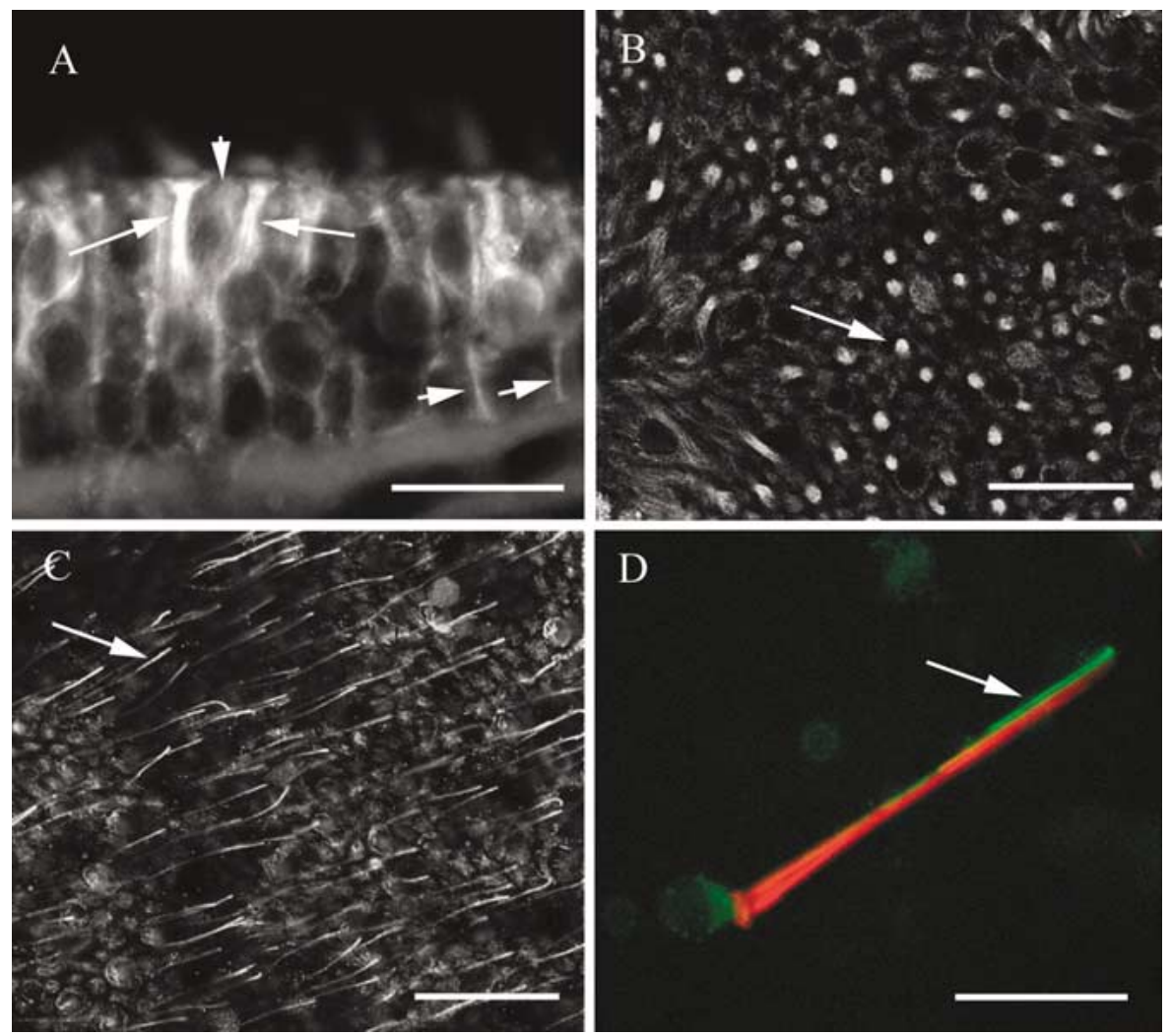

FIG. 4. $\beta_{\mathrm{IV}}$ tubulin in vestibular epithelia. A. Transverse section of a utricle showing bright label in the supranuclear regions of hair cells (long arrows) and in supporting cells (short arrows). B Confocal view of a utricle whole mount in a focal plane below the apex showing label in hair cells and supporting cells. The arrow indicates label in a hair cell surrounded by supporting cell label. Confocal image, optical section thickness-0.53 $\mu \mathrm{m}$. C Apical confocal view of a utricle whole mount showing label in kinocilia (one of which is indicated by the arrow), in hair cells, and in supporting cells. Optical section thickness-0.53 $\mu \mathrm{m}$. D Isolated vestibular hair cell showing label for actin (phalloidin-red) in stereocilia and the cuticular plate, and label for $\beta_{\mathrm{IV}}$ tubulin (green) in the soma and in the kinocilium. The scale bars represent $8 \mu \mathrm{m}$ for $\mathrm{A}$ and D, $25 \mu \mathrm{m}$ for B and C.
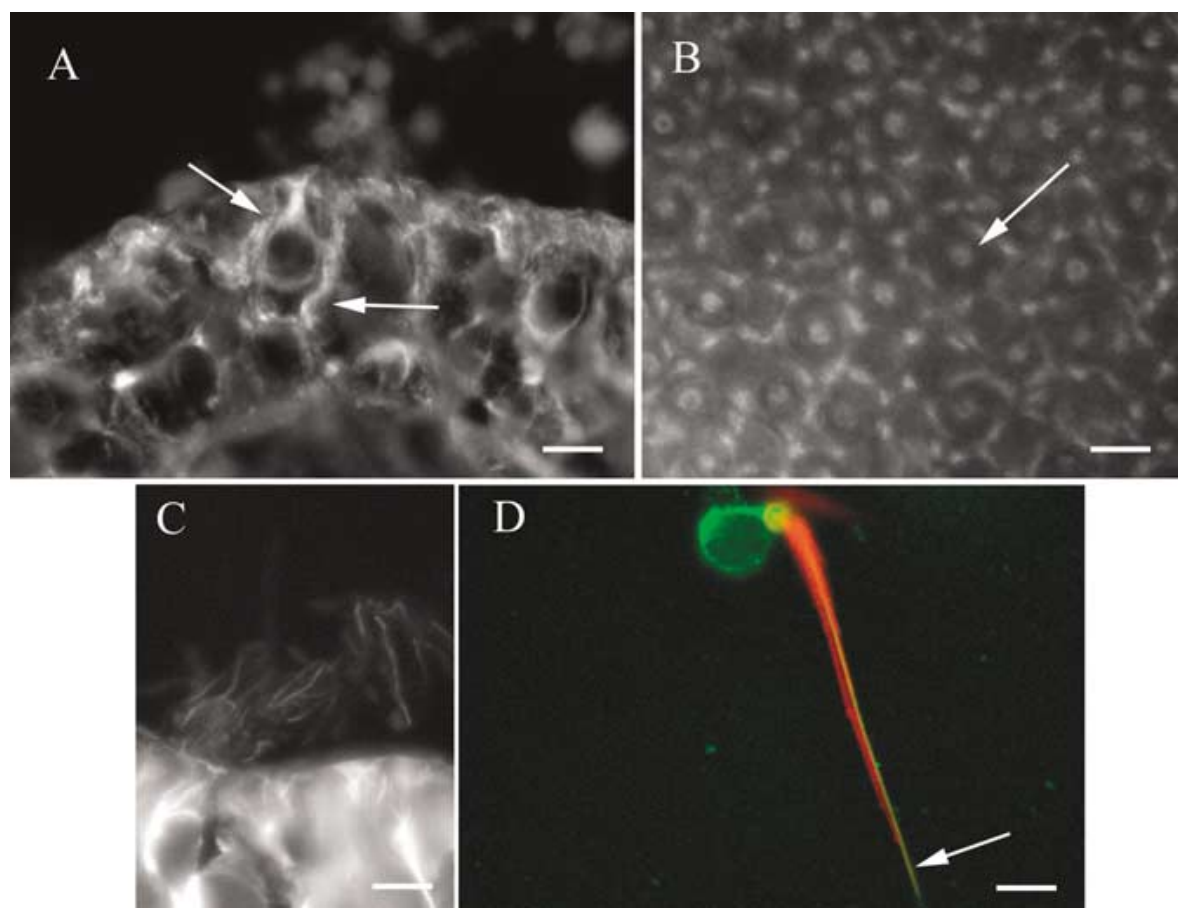

FIG. 5. $\quad \beta_{1}$ tubulin in vestibular epithelia. A Section of a crista showing label in a hair cell and a supporting cell (arrows). B Whole mount of a utricular macula showing patches of label in supporting cells surrounding label in a hair cell (arrow). C. Section of a crista showing labelin kinocilia. D Isolated vestibular hair cell showing label for actin (phalloidin-red) in stereocilia and the cuticular plate and label for $\beta_{1}$ tubulin (green) in the soma and in the kinocilium (arrow). The scale bar represents $8 \mu \mathrm{m}$ for $\mathrm{A}, \mathrm{B}$, and D and $7 \mu \mathrm{m}$ for $\mathrm{C}$.

contrast to the pattern seen for $\beta_{\text {IV }}$ tubulin, which was more prominent in hair cells. Supporting cell label in sections resembled that observed for $\beta_{\text {II }}$ tubulin. In whole mounts, the pattern again resembled that seen for $\beta_{\mathrm{IV}}$ tubulin. Figure 5B shows label in a utricular macula, visualized at the apical surface. Small, elon- gated patches of label were grouped in a circle, much as was found for $\beta_{\mathrm{II}}$ tubulin, but at the center of the circle was a bright patch of label, presumed to be the supranuclear microtubules of the hair cell.

$\beta_{\text {I }}$ tubulin was also present in vestibular hair cell kinocilia, as shown in Figure 5C, a section of a crista. 


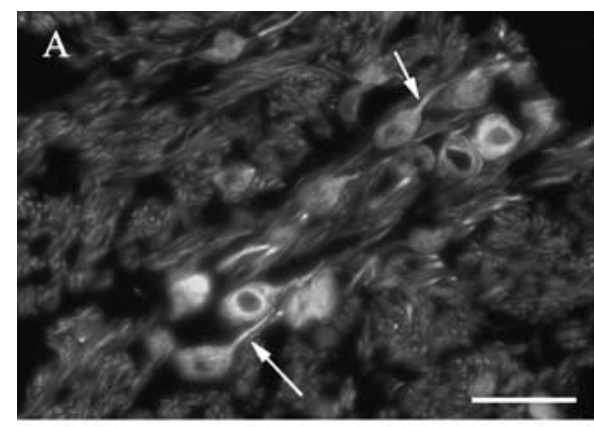

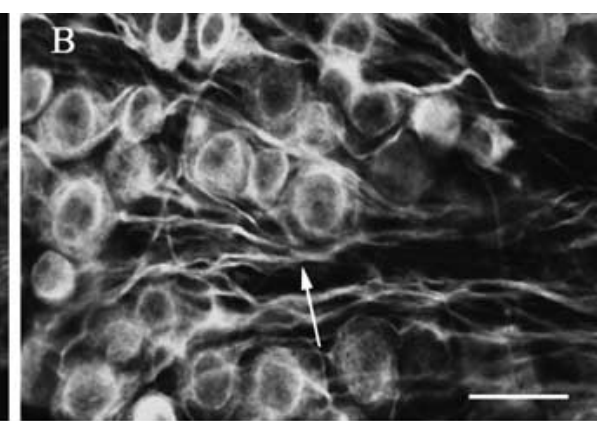

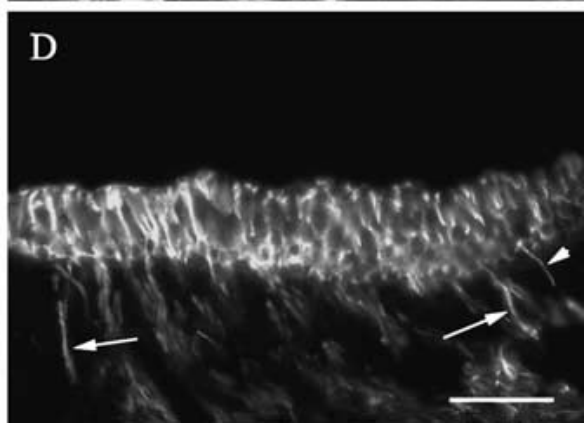

FIG. 6. Labeling for $\beta$ tubulin isotypes in vestibular ganglion cells. A Label for $\beta_{\text {III }}$ tubulin in somas and axons (arrows) of vestibular ganglion cells. B. Label for $\beta_{\text {II }}$ tubulin in somas and axons (arrows) of vestibular ganglion cells. Confocal image, optical section thickness-0.61 $\mu \mathrm{m}$. C Label for $\beta_{1}$ tubulin in dendrites of vestibular ganglion cells close to the junction between the horizontal and superior semicircular canal ampullary branches of the vestibular nerve. The somas of the ganglion cells are to the right of this image. Paired short arrows indicate label surrounding one dendrite. Confocal image, optical section thickness- $0.61 \mu \mathrm{m}$. D. Transverse section of a macula labeled for $\beta_{\mathrm{II}}$ tubulin showing label in largediameter axons (large arrows) and smalldiameter axons (small arrows). The scale bars represent $20 \mu \mathrm{m}$ for $\mathrm{A}$ and $\mathrm{D}$ and 25 $\mu \mathrm{m}$ for $\mathrm{B}$ and $\mathrm{C}$.
Labeled kinocilia could be seen as fine projections from the apical surface of hair cells in sections or as fine processes emanating from the apical surface of hair cells in whole mounts. In order to confirm the label in kinocilia, isolated vestibular hair cells were prepared and labeled for both $\beta_{\mathrm{I}}$ tubulin and actin using fluorescently labeled phalloidin. As shown in Figure 5D, hair cells were labeled in the soma and kinocilium for $\beta_{\mathrm{I}}$ tubulin, indicated in the figure in green. The single-labeled kinocilium is indicated by the arrow and is adjacent to the stereocilia bundle, which is indicated by the phalloidin label (red).

\section{Vestibular ganglion cells}

The somas of vestibular ganglion cells were strongly labeled by the antibodies for $\beta_{\mathrm{I}}, \beta_{\mathrm{II}}$, and $\beta_{\mathrm{III}}$ tubulin but not by the antibody for $\beta_{\mathrm{IV}}$ tubulin. Figure $6 \mathrm{~A}$ shows label for $\beta_{\text {III }}$ tubulin in ganglion cells in a section, showing label in somas and axons. The label in axons is particularly prominent. Figure $6 \mathrm{~B}$ shows similar label for $\beta_{\mathrm{II}}$ tubulin in a whole mount of the vestibular ganglion. Similar results were obtained with the antibody to $\beta_{\mathrm{I}}$ tubulin.

More peripherally, little or no label was seen in vestibular ganglion cell dendrites, except very close to the sense organs. Instead, label was seen in the form of parallel tracks surrounding the dendrite and therefore is presumed to be in the myelin sheaths of the dendrites rather than in the dendrites themselves. Figure $6 \mathrm{C}$ shows the labeling pattern for $\beta_{\mathrm{I}}$ tubulin in dendrites of vestibular ganglion cells at the bifurcation close to the horizontal and superior semicircular canal ampullae. The paired arrows show label surrounding a single large-diameter dendrite.
Similar patterns of label were seen for $\beta_{\text {II }}$ and $\beta_{\text {III }}$ tubulin, but no label was seen for $\beta_{\mathrm{IV}}$ tubulin in peripheral dendrites. However, close to both macula and ampullary sense organs, label was observed filling dendrites of varying diameters, stopping at the basal lamina. Figure $6 \mathrm{D}$ shows label for $\beta_{\mathrm{II}}$ tubulin in large-diameter and small-diameter dendrites. Similar label in terminal dendrites was found for $\beta_{\mathrm{I}}$ and $\beta_{\mathrm{II}}$ tubulin. As indicated in this figure, large-diameter dendrites tended to be closer to the middle of the organ (both ampullas and maculas), while small-diameter dendrites were more often seen toward the margin of the organs. It was not possible, in $\beta_{\mathrm{I}}$ and $\beta_{\text {II }}$ tubulin preparations, to follow the processes once they penetrated the basal lamina. $\beta_{\mathrm{IV}}$ tubulin was not observed anywhere in dendritic processes.

We have already seen the label for $\beta_{\text {III }}$ tubulin in vestibular epithelia as it forms calyceal endings surrounding hair cells (refer to Fig. 3). However, as is clear from the previous description of label patterns in vestibular epithelia, no similar label for $\beta_{\mathrm{I}}$ or $\beta_{\mathrm{II}}$ tubulin was found in calyceal nerve endings. We must conclude that only $\beta_{\mathrm{III}}$ tubulin is found in calyceal nerve endings.

\section{DISCUSSION}

The results of this study are illustrated in Figure 7 .

$\beta$ tubulin isotypes are selectively expressed in vestibular sensory epithelial cells but are not sorted into compartments within those cells

We observed $\beta_{\mathrm{I}}$ and $\beta_{\mathrm{IV}}$ tubulin in both the somas and the kinocilia of hair cells. The distributions of the two 


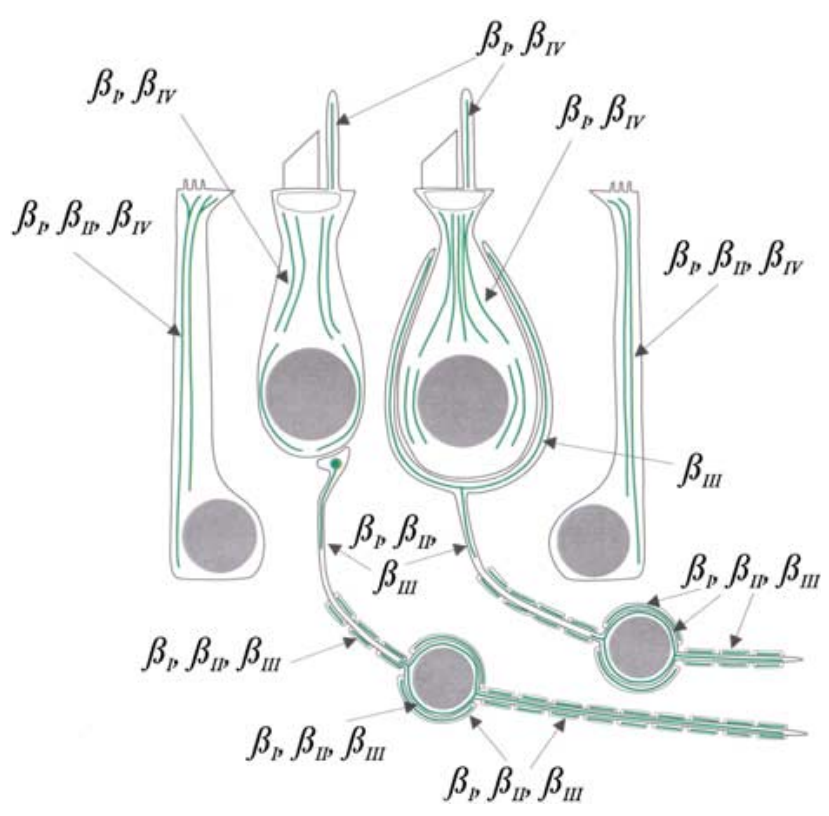

FIG. 7. Diagram of the distribution of $\beta$ tubulin isotypes in vestibular epithelial cells and neurons, based on the results of this study. Type I hair cell right of center, type II hair cell left of center, macular supporting cell right, crista supporting cell left.

isotypes are apparently entirely overlapping, although $\beta_{\mathrm{IV}}$ was more prominent than $\beta_{\mathrm{I}}$. In supporting cells, we observed $\beta_{\mathrm{I}}, \beta_{\mathrm{II}}$, and $\beta_{\mathrm{IV}}$ tubulin, each extending from the basal layer of the epithelium to the apical tuft. $\beta_{\mathrm{IV}}$ was less prominent in supporting cells than $\beta_{\mathrm{I}}$ or $\beta_{\text {II }}$ tubulin. Both vestibular hair cells and supporting cells, therefore, selectively express $\beta$ tubulin isotypes but do not sort the isotypes into separate compartments. In that respect, our findings here are consistent with previous findings for cochlea hair cells and supporting cells (Hallworth and Ludueña 2000). However, in detail, the findings are different. Vestibular hair cells have the same isotype composition as outer hair cells ( $\beta_{\mathrm{I}}$ and $\beta_{\mathrm{IV}}$ tubulin) but not of inner hair cells $\left(\beta_{\mathrm{I}}\right.$ and $\left.\beta_{\mathrm{II}}\right)$. The isotype composition of vestibular supporting cells $\left(\beta_{\mathrm{I}}, \beta_{\mathrm{II}}\right.$, and $\beta_{\mathrm{IV}}$ tubulin) is identical to that of cochlear Deiters' cells but not to that of inner or outer pillar cells $\left(\beta_{\mathrm{II}}\right.$ and $\beta_{\mathrm{IV}}$ only).

$\beta_{\mathrm{I}}$ and $\beta_{\mathrm{IV}}$ tubulin were present in both hair cells and supporting cells, but in supporting cells we also observed the $\beta_{\mathrm{II}}$ isotype. Thus, $\beta_{\mathrm{II}}$ tubulin is the only isotype found only in supporting cells of mammalian vestibular epithelia. $\beta_{\text {II }}$ tubulin could therefore be useful as a marker for supporting cells in mammalian hair cell regeneration studies, similar to the supporting cell antigen (Kruger et al. 1999) in avian hair cell regeneration studies.

All hair cells were labeled by the antibodies to $\beta_{\mathrm{I}}$ and $\beta_{\text {IV }}$ tubulin, although some hair cells were noticeably fainter than others. Type I hair cells have the highest concentration of microtubules, predomi- nantly in the supranuclear neck region (Favre and Sans 1983; Kikuchi et al. 1991). According to Kikuchi et al. (1991), these number 130-230 microtubules per cell in the guinea pig. In contrast, the reported 100-160 microtubules in the supranuclear region of type II hair cells are more diffuse (Kikuchi et al. 1991). It is likely, therefore, that the most brightly labeled hair cells in our preparations are type I. The more diffusely labeled hair cells may be considered to be type II hair cells. It is important to note, however, that type I and type II hair cells express the same two $\beta$ tubulin isotypes.

Our observation that $\beta_{\mathrm{III}}$ tubulin is not expressed in vestibular hair cells or supporting cells is also consistent with previous observations that $\beta_{\text {III }}$ is not expressed in hair cells or supporting cells of the mammalian cochlea (Hallworth and Ludueña 2000). In contrast, Stone and Rubel (2000) reported $\beta_{\text {III }}$ tubulin in both mature and regenerating chick cochlea hair cells. The absence of $\beta_{\text {III }}$ tubulin in any cell type other than neurons in vestibular epithelia corresponds to previous observations, using other antibodies, that $\beta_{\text {III }}$ tubulin is rarely expressed in other cell types (Burgoyne et al. 1988; Joshi and Cleveland 1989). Indeed, $\beta_{\text {III }}$ has been termed the "neuronspecific" tubulin for that reason, although $\beta_{\text {III }}$ tubulin has also been reported in colon (Roach et al. 1998).

$\beta$ tubulin isotypes are selectively expressed in vestibular neurons and are sorted into compartments within some of those cells

In this article we have shown that vestibular afferent neurons express the $\beta_{\mathrm{I}}, \beta_{\mathrm{II}}$, and $\beta_{\mathrm{III}}$ isotypes. The $\beta_{\mathrm{IV}}$ isotype was not observed in vestibular nerve, which is consistent with previous observations that $\beta_{\text {IV }}$ makes up only a small percentage of $\beta$ tubulin in brain (Banerjee et al. 1988).

Our observation that $\beta_{\text {III }}$ tubulin is compartmentalized to calyceal endings is unusual and interesting. It implies that there are mechanisms for sorting or localized expression of the isotype in a specific compartment of some neurons. It is possible that $\beta_{\mathrm{I}}$ tubulin is also present in calyceal endings but was obscured by all the other label in surrounding hair cells and supporting cells. However, inspection of Figure 2 should convince the reader that $\beta_{\text {II }}$ tubulin is not present in calyceal nerve endings.

The isotype content of kinocilia depends partly on the cell type

The kinocilia of vestibular hair cells are a tubulinbased axoneme-like organelle. Unlike other axoneme-based structures, such as the kinocilia of sperm or of respiratory epithelial cells, they are not motile. 
$\beta_{\text {IV }}$ tubulin is a consistent feature of kinocilia. In this study and a companion study (Woo et al. 2002), we have now added to the information available on the expression of $\beta$ tubulin isotypes in kinocilia. Woo et al. (2002) found that all four $\beta$ tubulin isotypes were found in the kinocilia of olfactory sensory neurons, while only two isotypes ( $\beta_{\mathrm{I}}$ and $\beta_{\mathrm{IV}}$ tubulin) were found in the kinocilia of respiratory epithelial cells. In both cases, as in the vestibular system, there was no difference between the isotypes found in kinocilia and the rest of the cell. However, Woo et al. (2002) reported that olfactory basal cells, the stem cells of olfactory neurons that have no olfactory cilia, expressed only $\beta_{\mathrm{I}}, \beta_{\mathrm{II}}$, and $\beta_{\mathrm{III}}$ tubulin. The expression of $\beta_{\mathrm{IV}}$ tubulin in olfactory neurons thus appears to accompany the development of olfactory cilia. It appears that kinocilia are assembled in an indiscriminant fashion from whatever isotypes are expressed by the cell but that $\beta_{\mathrm{IV}}$ tubulin is required.

\section{Selective expression of $\beta$ tubulin isotypes is common}

Our observation that cells express only selected $\beta$ tubulin isotypes is not new. Hallworth and Ludueña (2000), using the same antibodies as in this study, showed that the $\beta$ tubulin isotypes are differentially expressed in cochlear hair cells and supporting cells. Further, Roach et al. (1998), also using the same antibodies, demonstrated selective expression of isotypes in a variety of tissues. More recently, Woo et al. (2002) demonstrated a striking differential expression of isotypes in the olfactory system: Olfactory sensory neurons express all four isotypes, while the adjacent respiratory epithelial cells express only $\beta_{\mathrm{I}}$ and $\beta_{\mathrm{IV}}$ tubulin.

Others have shown differential expression of isotypes in different systems. Burgoyne et al. (1988) found that in rat cerebellum some isotypes are expressed in neurons, others in non-neuronal cells, still others in both cell types. Wang et al. (1986) studied two mouse isotypes, termed $\mathrm{M} \beta 1$ and $\mathrm{M} \beta 3$. Expression of $\mathrm{M} \beta 1$ was restricted to tissues involved in hematopoeisis (bone marrow, liver, lung, and spleen) and was not found in brain. Thus, selective expression of $\beta$ tubulin isotypes is common.

\section{Sorting of $\beta$ tubulin isotypes to within-cell fools} is rare

Our observation that at least some vestibular ganglion cells sort a particular isotype ( $\beta_{\text {III }}$ tubulin) to a particular compartment (the calyceal nerve endings) is unusual but not without precedent. Arai and Matsumoto (1988), observed two isotypes in the cell body of squid neurons but only one in the axon. Joshi and Cleveland (1989) found that $\beta_{\mathrm{II}}$ and $\beta_{\mathrm{III}}$ tubulin accumulated in PC-12 cells as a consequence of stimulated neurite outgrowth. Falconer et al. (1992) found that $\beta_{\mathrm{II}}$ tubulin is preferentially incorporated into colchicine-stable microtubules during development of embryonal carcinoma cells into neurons and muscle cells, while $\beta_{\text {III }}$ is preferentially incorporated into colchicine-labile microtubules. Renthal et al. (1993) found that the cilia of bovine rod outer segments and respiratory epithelia were enriched in $\beta_{\text {IV }}$ compared with $\beta_{\text {II }}$ and $\beta_{\text {III }}\left(\beta_{\text {I }}\right.$ was not examined). Perhaps the most striking example of segregated expression was found in Drosophila spermatogenesis (Nielsen et al. 2001). Axonemes and basal bodies utilize the same $\alpha$ tubulin but the $\beta 1$ isotype is found in the basal body while the $\beta 2$ isotype is found in the axoneme.

However, the most common finding is that $\beta$ tubulin isotypes are not compartmentalized, that is, all cellular microtubules contain all available isotypes (Lewis and Cowan 1988; Hallworth and Ludueña 2000; Woo et al. 2002). Furthermore, ectopic isotypes introduced into cells are incorporated uniformly into all microtubules (Joshi et al. 1987).

\section{The isotypes selected for expression may influence function}

The multi-tubulin hypothesis suggests that the small amino acid sequence differences between $\beta$ tubulin isotypes have been conserved for functional reasons. However, it has been difficult to ascribe specific functions to isotypes. $\beta_{\mathrm{IV}}$ tubulin appears to be prominent in cilia (Renthal et al. 1993; Lu et al. 1998; Roach et al. 1998) and also appears to interact with actin stress fibers (Walss-Bass et al. 2001). Changing the isotypes that a cell expresses should have functional consequences, and sometimes this can be shown to be true. In Drosophila, a moth testes-specific $\beta 2$ isotype resulted in the formation of 16 protofilament microtubules characteristic of the moth (Raff et al. 1997). In addition, overexpression of the Drosophila $\beta 1$ isotype results in axonemes with ten doublets instead of nine (Raff et al. 2000). Thus, the nature and quantity of isotypes available can influence microtubule architecture in an invertebrate. It remains to be demonstrated that $\beta$ tubulin isotypes may influence function in vertebrates.

\section{ACKNOWLEDGMENTS}

Supported by NIH grant CA26376, U.S. Army grant DAMD17-98-1-8246, and Welch Foundation grant AQ-0726 to R.F.L., and NIH grant DC02053 to R.H. We thank Joseph Kaeller for technical assistance and Tom Adrian, Xinquan 
$\mathrm{Li}$, and Xianzhong Ding for assistance with the Western blots. Purchase of the confocal microscope used in this study was made possible by grants from the Taub Foundation and the Nebraska Health Futures Foundation. We thank Tama Hasson for the myosin VIIA antibody.

\section{REFERENCES}

Arai T, Matsumoto G. Subcellular localization of functionally differentiated microtubules in squid axons: Regional distribution of microtubule-associated proteins and $\beta$-tubulin isotypes. $\mathrm{J}$. Neurochem. 51:1825-1838, 1988.

Banerjee A, Roach MC, Wall KA, Lopata MA, Cleveland DW, LUDUEÑA RF. A monoclonal antibody against the type II isotype of $\beta$-tubulin. Preparation of isotypically altered tubulin. J. Biol. Chem. 263:3029-3034, 1988.

Banerjee A, Roach MC, Trcka P, Ludueña RF. Increased microtubule assembly in bovine brain tubulin lacking the type III isotype of $\beta$-tubulin. J. Biol. Chem. 265:1794-1799, 1990.

Banerjee A, Roach MC, Trcka P, Ludueña RF. Preparation of a monoclonal antibody specific for the class IV isotype of $\beta$-tubulin. Purification and assembly of $\beta_{\mathrm{II}}, \beta_{\mathrm{III}}$, and $\beta_{\mathrm{IV}}$ tubulin dimers from bovine brain. J. Biol. Chem. 267:5625-5630, 1992.

Burgoyne RD, Cambray-Deakin MA, Lewis SA, Sarkar S, Cowan NJ. Differential distribution of $\beta$-tubulin isotypes in cerebellum. EMBO J. 7:2311-2319, 1988.

Falconer MM, Echeverri CJ, Brown DL. Differential sorting of beta tubulin isotypes into colchicine-stable microtubules during neuronal and muscle differentiation of embryonal carcinoma cells. Cell Motil. Cytoskel. 21:313-325, 1992.

FaVre D, Sans A. Organization and density of microtubules in the vestibular sensory cells in the cat. Acta Otolaryngol. 96:15-20, 1983.

Fulton C, Simpson PA. Selective synthesis and utilization of flagellar tubulin. The multi-tubulin hypothesis. In: Goldman R, Pollard J, Rosenbaum J (eds) Cell Motility, vol. 3. Cold Spring Harbor Laboratory Press, New York, pp 987-1005, 1976.

Gozes I, Barnstable CJ. Monoclonal antibodies that recognize discrete forms of tubulin. Proc. Natl. Acad. Sci. USA 79:25792583, 1982.

Hallworth R, Ludueña RF. Differential expression of $\beta$ tubulin isotypes in the adult gerbil organ of Corti. Hear. Res. 148:161$172,2000$.

Hallworth R, McCoy M, Polan-Curtain J. Tubulin expression in the developing gerbil organ of Corti. Hear. Res. 139:31-41, 2000.

Hasson T, Heintzelman MB, Santos-Sacchi J, Corey DP, Mooseker MS. Expression in cochlea and retina of myosin VIIa, the gene product defective in Usher syndrome type 1b. Proc. Natl. Acad. Sci. USA 92:9815-9819, 1995.

Jaeger RG, Fex J, Kachar B. Structural basis for mechanical transduction in the frog vestibular sensory apparatus: II. The role of microtubules in the organization of the cuticular plate. Hear. Res. 77:207-215, 1994.

Joshi H, Cleveland DW. Differential utilization of $\beta$-tubulin isotypes in differentiating neurites. J. Cell. Biol. 109:663-673, 1989.

Joshi HC, Yen TJ, Cleveland DW. In vivo coassembly of a divergent $\beta$ tubulin subunit (c $\beta 6$ ) into microtubules of different function. J. Cell Biol. 105:2179-2190, 1987.
Kikuchi T, Takasaka T, Tonosaki A, Watanabne H, Hozawa K, ShinkaWa H, Wada H. Microtubule subunits of guinea pig vestibular epithelial cells. Acta Otolaryngol. Suppl. 481:107-111, 1991.

Kruger RP, Goodyear RJ, Legan PK, Warchol ME, Raphael Y, Cotanche DA, Richardson GP. The supporting-cell antigen: A receptor-like protein tyrosine phosphatase expressed in the sensory epithelia of the avian inner ear. J. Neurosci. 19:48154827, 1999.

LEWIS SA, CowAN NJ. Complex regulation and functional versatility of mammalian $\alpha$ and $\beta$-tubulin isotypes during the differentiation of testis and muscle cells. J. Cell Biol. 106:2023-2033, 1988.

Lee MK, Tuttle JB, Rebhun LI, Cleveland DW, Frankfurter A. The expression and posttranslational modification of a neuron-specific $\beta$-tubulin isotype during chick embryogenesis. Cell Motil. Cytoskel. 17:118-132, 1990.

Lu Q, Moore GD, Walss C, Ludueña RF. Structural and functional properties of tubulin isotypes. Adv. Struct. Biol. 5:203-227, 1998.

Ludueña RF. The multiple forms of tubulin: Different gene products and covalent modifications. Int. Rev. Cytol. 178:207-275, 1998.

Maue RA, Dionne VE. Preparation of isolated mouse olfactory receptor neurons. Pflügers Arch. 409:244-250, 1987.

Nielsen MG, Turner FR, Hutchens JA, Raff EC. Axoneme-specific $\beta$-tubulin specializations: A conserved C-terminal motif specifies the central pair. Curr. Biol. 11:529-533, 2001.

Ogata Y, Slepecky NB. Immunocytochemical comparison of posttranslationally modified forms of tubulin in the vestibular endorgans of the gerbil: tyrosinated, acetylated and polyglutamylated tubulin. Hear. Res. 86:125-131, 1995.

Raff EC, Fackenthal JD, Hutchens Ja, Hoyle HD, Turner FR. Microtubule architecture specified by a $\beta$-tubulin isoform. Science 275:70-73, 1997.

Raff EC, Hutchens JA, Hoyle HD, Nielsen MG, Turner FR. Conserved axoneme symmetry altered by a component $\beta$-tubulin. Curr. Biol. 10:1391-1394, 2000.

Renthal R, Schneider BG, Miller MA, Ludueña RF. $\beta_{\text {IV }}$ is the major $\beta$-tubulin isotype in bovine cilia. Cell Motil. Cytoskel. 25:19-29, 1993.

Roach MC, Boucher VL, Walss C, Ravdin PM, Ludueña RF. Preparation of a monoclonal antibody specific for the class I isotype of $\beta$-tubulin: The $\beta$ isotypes of tubulin differ in their cellular distributions within human tissues. Cell Motil. Cytoskel. 39:273285, 1998.

Stone JS, Rubel EW. Temporal, spatial, and morphologic features of hair cell regeneration in the avian basilar papilla. J. Comp. Neurol. 417:1-16, 2000.

Walss-Bass C, Prasad V, Kreisberg Ji, Ludueña RF. Interaction of the $\beta_{\mathrm{IV}}$-tubulin isotype with actin stress fibers in cultured rat kidney mesangial cells. Cell Motil. Cytoskel. 49:200-207, 2001.

Wang D, Villasante A, Lewis SA, Cowan NJ. The mammalian $\beta$ tubulin repertoire: Hematopoietic expression of a novel, heterologous $\beta$-tubulin isotype. J. Cell. Biol. 103:1903-1910, 1986.

Woo K, Jensen-Smith HC, Ludueña RF, Hallworth R. Differential expression of $\beta$ tubulin isotypes in gerbil nasal epithelia. Cell Tissue Res. 309:331-335, 2002. 